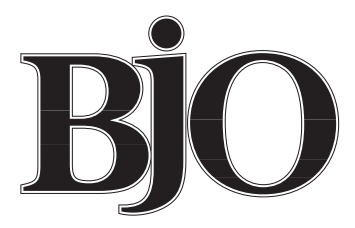

British Journal of Ophthalmology

\title{
Editorials
}

\section{Disease and "dis-ease" in patients with uveal melanoma}

Patients with uveal melanoma have plenty to worry about-loss of vision, pain, loss of the eye, and death from metastatic disease, not to mention all the secondary consequences of these problems. Previously, all we had to do was to remove the threat of metastatic disease by enucleation. Recently, we have been expected to conserve the eye with as much vision as possible. Nowadays, success also depends on how well we are able to restore the patient's peace of mind (and rightly so).

As always, what really matters in the end is that the patient is happy with the result. We have learnt the hard way that expensive technical excellence may be a total waste of precious time and energy if the patient is seriously inconvenienced by metamorphopsia or worried about the possibility of local tumour recurrence. We now appreciate that with a newly diagnosed patient our first priority is to get to know what that patient really wants and needs, what degree of risk that patient would feel comfortable with, and what commitment that patient is prepared to make to achieve a particular goal. It is not simply a question of deciding between enucleation and conservative treatment but more often a matter of selecting between radiotherapy and local resection or between radiotherapy and phototherapy.

Gone are the days when we intuitively selected a treatment, which was then imposed on the patient. Now, quite appropriately, we are expected to involve the patient in the process of treatment selection and to base any decisions on sound scientific evidence. Until recently, when discussing various therapeutic options with our patients, all we had to do was to conjure up in our photographic memories various Kaplan-Meier survival curves depicting actuarial rates of visual loss, local tumour recurrence, enucleation, and metastatic disease. Nowadays, we must also be able to advise our patients on how they are likely to feel after treatment and how they may cope (or otherwise) if things don't quite go according to plan. This task might seem an ordeal to ophthalmologists who are not accustomed to dealing with cancer but to an ocular oncologist it can be quite an uplifting experience to act as a special guide, steering patients through turbulent times towards a new tranquillity.

When considering quality of life, we should increasingly be relying on hard psychological data gained by proper research. A vast number of "instruments" (that is, questionnaires) have been developed to measure how diseases and treatments influence the ways in which patients continue to survive physically, psychologically, and socially. Generic instruments, such as the EORTC QLQ-C30 and the Hospital Anxiety Depression (HAD) score, investigate general factors - that is, tiredness, anxiety, depression, family life, social activities, and financial difficulties. Disease specific instruments focus on particular conditions, such as the VF-14, which was developed to measure visual handicap caused by cataract. There is a tendency to use such "off the shelf" instruments for assessing patients with uveal melanoma. ${ }^{12}$ However, visual function is only one of many important outcomes in patients with this tumour and what is required is a dedicated instrument, tailormade to address the specific symptoms and difficulties caused by this particular disease. Such an instrument must also be shown to be user friendly in different languages and cultures, reliable, and sensitive to change in patient status. ${ }^{3}$ The Ophthalmic Oncology Group (OOG) of the European Organisation for Research and Treatment of Cancer (EORTC) is currently developing such an instrument. The study involves workers from different countries and is following strict guidelines of the EORTC's Quality of Life Study Group. The OOG questionnaire measures (1) comfort (for example, ocular grittiness, ache, watering, dryness, photophobia, headache); (2) vision (for example, blurring, metamorphopsia, visual field loss, diplopia, photopsia, floaters, interference with fellow eye); (3) specific worries (for example, loss of the eye, local tumour recurrence, metastatic disease, cosmetic appearance, regrets about treatment choice); and (4) visual handicap (for example, reading, driving, walking, pouring tea, etc). Preliminary studies by Damato and Humphreys in a sample of 240 patients with treated uveal melanoma showed reassuringly predictable results, but there were a few surprises. For example, patients were as likely to report fears of local recurrence after enucleation as after conservative therapy. A modified questionnaire is now routinely completed by every patient at each follow up visit and this is proving invaluable in identifying problems, many of which can be alleviated by simple reassurance. Such specific questioning is infinitely better than cheerfully asking "How's life?", which invariably elicits the polite response, "OK".

Quality of life studies must be interpreted with due caution. For example, when comparing quality of life after radiotherapy with that after enucleation, it is essential to ask the following questions: Are the samples large enough to recognise a statistical difference if there is one? Did the radiotherapy sample include patients who should really have had enucleation in the first instance? Did the questionnaires ask the right questions? What were the physical outcomes after radiotherapy? How many enucleated patients actually had any choice about their 
treatment? How applicable would the study be, say, to a young woman, an old man, or a patient with poor vision in the fellow eye? Assuming that the treatment was delivered competently, what could be the cause of poor quality of life after treatment? Could it be an unrelated condition (for example, arthritis), poor treatment selection, inadequate counselling, or a failure to identify and treat psychological problems? To some extent, a quality of life measurement reflects the performance of the entire tumour service and not just the treatments under investigation.

In conclusion, the management of patients with uveal melanoma should improve considerably if ocular symptoms and overall quality of life were measured systematically in all patients at regular intervals after treatment, using an internationally recognised instrument. The MOOD questionnaire described by Foss and colleagues in this issue of the $B \mathcal{F O}(\mathrm{p}$ 347) will in future be one of many and time will tell which of these will gain widespread acceptance.

BERTIL DAMATO

Ocular Oncology Service, St Paul's Eye Unit, Royal Liverpool

University Hospital, Prescot Street, Liverpool L7 8XP

1 Cruickshanks KJ, Fryback DG, Nondahl DM, et al. Treatment choice and quality of life in patients with choroidal melanoma. Arch Ophthalmol 1999;117:461-7.

2 Quality of Life Assessment in the Collaborative Ocular Melanoma Study. Design and methods. COMS-QOLS Report No 1. COMS Quality of Life Study Group. Ophthalmic Epidemiol 1999;6:5-17.

3 Maguire P, Selby P. Assessing quality of life in cancer patients. $\mathrm{Br}$ F Cancer $1989 ; 60: 437-40$

\section{Foveal translocation for exudative age related macular degeneration}

The idea that the macular neurosensory retina might be surgically detached and then reattached in such a manner that the fovea is relocated on the underlying retinal pigment epithelium (RPE) and choroid has been attributed to Lindsey et al. ${ }^{1}$ However, the 1983 ARVO abstract did not make clear that the main purpose of this exercise was to restore vision in eyes with subfoveal choroidal neovascular membranes (CNVMs) complicating age related macular degeneration (AMD), and the experimental and theoretical potential of the method was first realised clinically by Machemer and Steinhorst ${ }^{2}$ only in 1993. Their "macular rotation" procedure comprised induction of retinal detachment, vitrectomy and $360^{\circ}$ peripheral retinotomy, removal of the CNVM (and any associated subretinal haemorrhage), and $30-80^{\circ}$ rotation of the retina around the axis of the optic nerve before silicone oil injection and $360^{\circ}$ endolaser retinopexy. Of the three eyes so treated, one showed a major visual improvement while proliferative vitreoretinopathy (PVR) compromised the outcome in the other two eyes. Modifications of the macular rotation method then followed ${ }^{3-6}$ but only Eckardt and colleagues from Frankfurt ${ }^{6}$ have succeeded in perfecting the technique, including minimising PVR and simultaneously counterrotating the globe to avoid a tilted image. A majority of the 30 eyes which they treated in 1997-8 showed an improvement in distance acuity, an improvement in near acuity, and maintenance or restoration of comfortable reading vision after silicone oil removal and cataract surgery with a mean follow up of 10.5 months. Notwithstanding the absence of a control series and independent visual assessment, $25-50^{\circ}$ macular rotation for foveal relocation in AMD appears not only to have been validated, but also (given the difficulty and duration of the surgery) to have been vindicated by Eckardt's success. His results far exceed any remote expectation of spontaneous reversal of the generally progressive natural history of AMD (for example, improvement in distance acuity after 12 months in only $7 \%$ of the control group in another AMD study), ${ }^{7}$ and they provide a standard against which all future refinements of foveal relocation surgery must be judged. Eckardt's results also put into perspective the supposed merits of alternative therapies for subfoveal CNVMs in AMD for which randomised trials have demonstrated a statistically significant benefit. In the case of laser ablation of subfoveal CNVMs, ${ }^{8}$ for example, any prospect of future reading vision is instantly destroyed by the laser treatment, and the method has proved so intrinsically anti-Hippocratic ("first do no harm") as to render immaterial the evidence base of overall long term benefit, at least in the UK and Ireland. ${ }^{9}$ In the case of photodynamic therapy (PDT), ${ }^{7}$ the comparative figure for improvement in distance acuity 12 months after 3 monthly therapy using verteporfin is $16.4 \%$, the main benefit of PDT manifesting as a slowing of the rate of visual deterioration; no information is available on PDT concerning that most relevant of functional outcomes - reading capability. All the while we have also witnessed the false dawns created by other potential approaches to exudative AMD such as interferon, radiotherapy, and surgical excision of CNVMs.

The sustained benefits of macular rotation await confirmation through additional (and preferably properly controlled) series and extended follow up (especially to provide data on recurrence of CNVMs). However, work has been progressing in the meantime on exploring more practical approaches to foveal relocation. DeJuan and colleagues from the Wilmer Institute ${ }^{10}$ have described a method for obtaining limited foveal relocation (up to $15^{\circ}$ or $1.5 \mathrm{~mm}$ ) without recourse to peripheral retinotomy and thereby hopefully reducing the risk of PVR and obviating the need for extraocular muscle surgery. After inducing a retinal detachment in the vitrectomised eye posterior retinal redundancy is created by scleral shortening, and foveal relocation is induced by gas injection and postoperative posturing in a manner akin to that responsible for the macular folds which can complicate gas tamponade in externally buckled and vitrectomised eyes. ${ }^{11}$ Because forceps removal of a CNVM frequently involves extraction of an even wider area of RPE (D Wong, personal communication), the CNVM is left in situ and is destroyed by laser photocoagulation postoperatively provided the membrane is no longer subfoveal. Anecdotal reports of spectacular individual successes from such procedures have captured the imagination (not least of the tabloid press) and a relocation of $0.5 \mathrm{~mm}$ has also been achieved by the same method but without recourse to scleral shortening. ${ }^{12}$ However, the overall outcomes ${ }^{13}$ from this technique have so far been disappointing, apparently reflecting (a) the difficulty in predicting the potential for visual recovery in individual cases (presumably depending on the 
degree of secondary photoreceptor apoptosis preoperatively and the health of the RPE), (b) the complications of postoperative photocoagulation (not least subfoveal recurrence) of the newly extrafoveal CNVM, and (c) the difficulty in predicting the precise location which the fovea will take up postoperatively. This last problem has now been addressed by Wong and Lois from Liverpool who report their results of direct intraoperative macular manipulation for foveal relocation in this issue of the $B F O(\mathrm{p} 352)$. A crucial element of their technique involves internal drainage of the subretinal infusate and patience in allowing the effect of the RPE pump to "kick in" to maintain the induced ectopia; two of the nine eyes in their series had useful reading vision 3 and 4 months after the foveal relocation. The significance to be attached to retention of vision and postsurgical reduction in vision in the other seven patients is difficult to judge in the absence of a control series but the authors' expectation of improved outcomes in the future with further refinement of the technique appears to be justified. Nevertheless, given the rapid pace of developments in foveal relocation surgery for exudative AMD, perhaps the time has now come for an international "tracker trial" 14 of this surgery and to echo the recommendation of DeJuan and colleagues ${ }^{10}$ that continued investigation of such techniques only occurs "in the context of a clinical research effort approved by an independent institutional review board".

Manchester Royal Eye Hospital
1 Lindsey P, Finkelstein D, D'Anna S. Experimental retinal rotation. Invest Ophthalmol Vis Sci (Suppl) 1983;24:242.

2 Machemer R, Steinhorst UH. Retinal separation, retinotomy, and macular relocation: II A surgical approach for age-related macular degeneration? Graefes Arch Clin Exp Ophthalmol 1993;231:635-41.

3 Ninomiya Y, Lewis JM, Hasegawa T, et al. Retinotomy and foveal translocation for surgical management subfoveal choroidal neovascular membranes. Am f Ophthalmol 1996;122:613-21.

4 Wolf S, Lappas A, Weinberger AWA, et al. Macular translocation for surgical management of subfoveal choroidal neovascularisations in patients with AMD: first results. Graefes Arch Clin Exp Ophthalmol 1999;237:51-7.

5 Akduman L, Karavellas MP, MacDonald JC, et al. Macular translocation with retinotomy and retinal rotation for exudative age-related macular degeneration. Retina 1999;19:418-23.

6 Eckardt C, Eckardt U, Conrad H-G. Macular rotation with and without counter-rotation of the globe in patients with age-related macular degeneration. Graefes Arch Clin Exp Ophthalmol 1999;237:313-25.

7 Treatment of Age-related Macular Degeneration with Photodynamic Therapy (TAP) Study Group. Photodynamic therapy of subfoveal choroidal neovascularisation in age-related macular degeneration with verteporfin. Arch Ophthalmol 1999;117:1329-45

8 Macular Photocoagulation Study Group. Visual outcome after laser photocoagulation for subfoveal neovascularisation secondary to age-related coagulation for subfoveal neovascularisation secondary to age-related macular degeneration. The influence of initi
acuity. Arch Ophthalmol 1994;112:480-8.

9 Beatty S, Au Eong KG, McLeod D, et al. Photocoagulation of subfoveal choroidal neovascularisation in age-related macular degeneration: the impact of the macular photocoagulation study in the United Kingdom and Republic of Ireland. Br f Ophthalmol 1999;83:1103-4.

10 DeJuan E, Loewenstein A, Bressler NM, et al. Translocation of the retina for management of subfoveal choroidal neovascularisation. II: A preliminary report in humans. Am $\mathcal{F}$ Ophthalmol 1998;125:635-46.

11 Twomey JM, Leaver PK. Retinal compression folds. Eye 1988;2:283-7.

12 DeJuan E, Vander JF. Effective macular translocation without scleral imbrication. Am f Ophthalmol 1999;128:380-2.

13 Lewis H, Kaiser PK, Lewis S, et al. Macular translocation for subfoveal choroidal neovascularisation in age-related macular degeneration: a prospective study. Am $\mathcal{F}$ Ophthalmol 1999;128:135-46.

14 Lilford RJ, Braunholtz DA, Greenhalgh R, et al. Trials and fast changing technologies: the case for tracker studies. BMF 2000;320:43-6. 